How to reference this article

Zaccaria, M. (2019). Diana, Aurelia e le altre: attrici e capocomiche dell'ultimo duca di Mantova. Italica Wratislaviensia, 10(2), 103-117.

DOI: http://dx.doi.org/10.15804/IW.2019.10.1.19

Michela Zaccaria

Università degli Studi di Firenze

michela.zaccaria@unifi.it

ORCID: 0000-0001-7224-6761

\title{
DIANA, AURELIA E LE ALTRE: ATTRICI E CAPOCOMICHE DELL'ULTIMO DUCA DI MANTOVA
}

\author{
DIANA, AURELIA AND THE OTHERS: ACTRESSES \\ AND LEADERS OF THE LAST DUKE OF MANTUA
}

\begin{abstract}
This article introduces the activity of artistic directors Teresa Costantini, Angela Paghetti, and Colomba Coppa in the service of Ferdinando Carlo Gonzaga-Nevers, their generous patron and the last Duke of Mantua. Referencing studies that were initiated 30 years ago in the field of musicology and using recent historical research and archival sources, the essay highlights the artistic, organisational, and management responsibilities of commedia dell'arte directors. It reconstructs their relations with Ferdinando Carlo, ducal agents, and the Gonzaga court, while also focussing on their relations with Venetian Grimani impresarios, with whom the Duke of Mantua had established a lasting and generous collaboration.

Performing women, prejudicially equated with prostitutes and beneficiaries of gifts and privileges, were depictions of love and glory for a discredited prince, whose array of artists was, however, amongst the most dense and dynamic in Italy. Actresses progressively moved away from the codes of courtliness and, as professionals registered and paid with money, they came to have strong bargaining power and levels of autonomy. However, they were subjected to rules and obligations, especially in Venice, where theatre owners made spaces available only to companies under the protection of the princes who were politically aligned with the Serenissima. During the years of the so-called Spanish succession war, Diana, Aurelia, and the others moved between Venice, Mantua, and Casale, where Ferdinando Carlo had moved after the blockade of Mantua, accompanied by a colourful procession of women, musicians, and commedia actors. Through theatrical misadventures mingled with military manoeuvres in Mantuan territory, and via changes of destination, programme, or repertoire, we read the story of a duchy that had lost its identity and of a rapidly changing theatrical market.
\end{abstract}

Keywords: actress, commedia dell'arte, Ferdinando Carlo Gonzaga-Nevers, leader, theatrical market 
1. La guerra di successione spagnola non impedì a Ferdinando Carlo Gonzaga-Nevers d'acquistarsi qualche credito come mecenate di artisti, sebbene politicamente naufragato da anni con la nomea di debosciato e di doppiogiochista. Aveva stipulato un trattato segreto di alleanza con le potenze borboniche, accogliendo a Mantova un presidio gallo-ispanico dall'aprile 1701. Leopoldo d'Austria lo aveva destituito del titolo, le truppe imperiali guidate da Eugenio di Savoia avevano cinto d'assedio la città e il duca era fuggito prima a Casale, poi in Francia.

Sin dal severo giudizio di Ludovico Antonio Muratori (Muratori, 1827, p. 80), l'ultimo duca di Mantova incarnava il cliché del principe vizioso, indegno epigono di un'illustre dinastia. Eccentrico e istrionico ${ }^{1}$, amava le donne e i cavalli di razza, ma le sue intemperanze ebbero un ruolo secondario nel fallimento della sua fortuna; al contrario, furono la mancanza di credibilità internazionale e la scelta di porsi al di fuori delle logiche di potere tra i piccoli potentati padani che determinarono la fine del suo ducato.

Ferdinando Carlo spendeva dissennatamente e la corte assomigliava a un grottesco serraglio, di cui facevano parte uno stuolo di cantanti e di attrici a sua disposizione in quel piccolo mondo creato a suo capriccio. Offriva loro un ottimo compenso, anticipava le spese del viaggio, talvolta concedeva l'uso di abitazione, le dotava di un passaporto ducale che rispondeva a una casistica complicata (Besutti, 1989, p. 13; Cirani, 2004, p. 45). Si attardava con loro dietro le quinte e alcuni intrattenimenti privati pare assomigliassero più a orge che a spettacoli ${ }^{2}$. Delle sue artiste era geloso e quando nel 1685 una delle sue più famose canterine, Margherita Salicola, gli preferì (in tutti i sensi) l'elettore di Sassonia Giovan Giorgio III, Ferdinando Carlo aprì un vero e proprio caso diplomatico ${ }^{3}$. Tuttavia l'ultimo Gonzaga non si sottraeva alla consuetudine di Antico

1 Cfr. Fochessati, 1930, p. 207 dove si riporta il giudizio del residente Breteuil su «le goût des mises ridicules et des allures théâtrales» del duca.

2 «Le duc de Mantoue ne s'acquiert pas ici beaucoup d'estime. Il est ordinaire avec les courtisanes, et à la comédie il se tient plus derrière le théâtre avec les comédiennes que dans sa loge» (conte d'Avaux, ambasciatore del Re Sole, 1673, citato in Fochessati, 1930, p. 195 e in Selfridge-Field, 1998, p. 220).

3 Rimando a Diari Legatizi (1685) riportati in Ricci, 1888, pp. 358-359n. 
Regime di prestare o di scambiare solisti e organici con altri principi. I vincoli professionali cui sottoponeva cantanti e attrici famose variavano da un caso all'altro. Da una parte, costoro erano beneficiarie di regali e di privilegi e potevano dichiararsi esponenti della corte gonzaghesca; dall'altra, offrendo la loro opera anche altrove e ricevendo una retribuzione in denaro, erano soprattutto professioniste scritturate, impegnate perlopiù sul versante del teatro a gestione impresariale.

In Laguna i proprietari dei teatri resero disponibili i luoghi di spettacolo esclusivamente alle compagnie sotto la protezione dei prìncipi politicamente allineati con la Serenissima (ad esempio Parma o Mantova) (Selfridge-Field, 1998, p. 212). Qui Ferdinando Carlo stabilì una proficua collaborazione con Giovan Carlo e Vincenzo Grimani dei teatri San Giovanni Grisostomo e San Samuele e legati a lui da vincoli di parentela ${ }^{4}$, anche dopo il loro accordo con i Vendramin del teatro San Luca, che monopolizzò il mercato teatrale veneziano. I Grimani, di simpatie filoimperiali, erano in difficoltà a reclutare artisti provenienti dai vicini potentati sotto l'influenza francese e il duca mantovano li provvide di scritturati. In particolare, il Gonzaga-Nevers ebbe significativi legami con l'opera e la schiera dei suoi virtuosi fu tra le più folte e dinamiche, come prova la consuetudine di identificarli nei libretti attraverso la loro filiazione alla corte di questo principe. Gli artisti sotto la sua protezione moltiplicarono le attività fra Mantova, Venezia - dove il duca soggiornava durante il Carnevale - e Casale, residenza dove si trasferì dopo il blocco di Mantova con un variopinto corteggio di donne, musicisti e commedianti. Il suo gusto in fatto di spettacoli era probabilmente indiscriminato, ma non indifferente alla qualità delle performances di ottimo livello. Ferdinando Carlo si occupava personalmente della formazione delle compagnie e riceveva suppliche e richieste da singoli artisti e dal capocomico, ruolo che (come noto) poteva essere rivestito anche da una donna. A negoziare pensavano gli agenti ducali: Giovanbattista

${ }^{4}$ La madre Elena era la figlia di Ludovico Francesco Gonzaga, quarto marchese di Palazzolo. Vincenzo Grimani (1655-1710) si allineò con l'impero e i Savoia, poiché era amico personale del principe Eugenio. Fu fatto cardinale nel 1697, rappresentando l'imperatore in Roma durante la guerra di successione spagnola; dal 1708 fu viceré di Napoli. Cfr. Borrelli, 2002 e Selfridge-Field, 1998, p. 217. 
Pico duca di Mirandola trattava le faccende di Casale, il conte Lorenzo Beretti era coinvolto nel procurare artisti a Venezia, il conte Carlo Maria Vialardi ${ }^{5}$, influente ministro e consigliere di stato, si interessava del teatro mantovano.

2. All'inizio del Settecento le troupes sotto l'egida del Gonzaga-Nevers erano tutte dirette da donne: Teresa Costantini, Colomba Coppa e Angela Paghetti di stanza a Venezia presso il Teatro San Luca (dei Vendramin) e il San Samuele (dei Grimani); Anna Marini a Mantova nel teatro detto «delle Commedie» nelle adiacenze dell'attuale Piazza Arche ${ }^{6}$.

Le capocomiche organizzavano le tournées e discutevano i contratti, decidevano la distribuzione delle parti nelle opere nuove e l'occorrente per la messinscena (attrezzeria, costumi), concertavano le scene comiche con le maschere. Il duca dava garanzie e faceva fronte alle inevitabili rivalità col tenere all'oscuro l'una degli affari dell'altra:

Vivo sempre con la memoria di ciò ch'il Serenissimo si degnò promettermi in Mantova, quando, onorandomi d'udienza, mi prese per la mano, e s'impegnò in parola di principe che mi avrebbe dato il contento d'una perfetta compagnia [...]. (ASMn, AG, E. XLV. 3, b. 1584) ${ }^{7}$

5 Carlo Maria Vialardi (?-1715) fu inviato ducale presso le corti di Vienna, Monaco, Dresda e Parma, fu governatore di Casale e Guastalla e ministro del ducato mantovano dal 1691. Insieme al fratello Romualdo, era estremamente influente: dal 1699 sovrintendente generale alle vie di comunicazione e dal 1703 nel consiglio di reggenza con l'incarico di amministrare il territorio in assenza del Gonzaga-Nevers (ASMn, ms. C. D'Arco, Notizie bibliografiche delle famiglie illustri mantovane. VII, p. 297 e Bianchi, 2012, pp. 156-162). Salvo diversa avvertenza, le fonti archivistiche registrate nel presente contributo sono inedite.

${ }^{6}$ Esperienze analoghe raccordano queste attrici al più ampio contesto del sistema teatrale italiano di fine Seicento. Solo a titolo di esempio, si pensi alle figure di Giulia De Caro a Napoli, di Agata Vitaliani Calderoni a Ferrara, di Marzia Narici Fiala a Modena. Tuttavia il ruolo capocomicale e impresariale delle donne attende a oggi uno studio più sistematico.

${ }^{7}$ Lettera di Teresa Corona Costantini a ignoto, Venezia, 18 novembre 1702, cc. n.n. La trascrizione è in gran parte conservativa, con sola normalizzazione delle maiuscole e, nel caso, della punteggiatura. 
Teresa Corona Costantini detta Diana ${ }^{8}$ apprezzava il gesto galante del duca. Era ancora avvenente, nonostante l'età, sebbene la sua bellezza nascondesse qualche piccolo segreto ${ }^{9}$. Attrice assai nota, sapeva cantare e suonare vari strumenti, ma era invisa ai colleghi per il pessimo carattere. Quando vent'anni prima furoreggiava sui palcoscenici veneziani nella compagnia finanziata da Alessandro Farnese, fratello del duca di Parma Ranuccio II, era invidiata anche per via di potenti protettori ${ }^{10}$ :

Gode questa signora un portamento così nobile et un gesto così proprio della scena che potria in questo genere dar le regole del vero rappresentare in palco alla Grecia che ne trovò l'invenzione. Si porta con tale disinvoltura, si veste così bene degl'affetti che esprime, che fa sospirar se si duole, semina riso se si rallegra ${ }^{11}$.

Con Mantova assediata dalle truppe imperiali, «si continuano nella città divertimenti di comedie, et altre allegrezze, ma però (...) c'è molta penuria $\rangle^{12}$. Teresa detta Diana se ne lamentava. Si era assunta un difficile compito e con audacia e testardaggine rivendicava l'impegno che il signore aveva preso con lei:

8 Teresa Corona (1660 circa-1730?), moglie di Giovanni Battista Costantini (Ottavio) dal quale ebbe almeno due figli, se ne separò lavorando al servizio dei Farnese, degli Este, dei Gonzaga-Nevers. Recitò negli intermezzi comici delle opere in musica al San Bartolomeo di Napoli (1710) e al Rangoni di Modena (1714). Cfr. Rasi, 18971905. II, pp. 733-734 e 759-762; Morinello, 1956 e Zaccaria, 2018.

9 «Fu Diana creduta una bellezza, fu amata, fu stimata, ma l'arti del sesso formavano in lei un incanto, che fu poi scoperto fallace. Basta il dire che i suoi denti, quali perle encomiati, non erano suoi, ma simulati, e posticci. Un accidente scoperse l'inganno, che fu a' suoi adoratori per molti lustri celato» (voce Diana in Bartoli, 1781-1782, pp. 204-206).

${ }^{10}$ Cfr. l'informazione «Fu Protetta da Personaggi di rango» (Ibidem) con «Fu cara a Principi», poi censurata con un tratto di penna dall'archivista ottocentesco in ASMn, Schede Davari, b. 14, Comici e Commedie (1408-1787), Diana e Silvio, c. 257r.-v.

${ }_{11}$ «Pallade Veneta. Novembre 1687» cui segue sonetto anonimo Scese dall'alto ciel l'offesa luna dedicato all'attrice, trascritti in Selfridge-Field, 1985, p. 196.

12 Lettera di Teresa Corona Costantini a ignoto, Venezia, 4 febbraio 1702 in Selfridge-Field, 1988, p. 225. 
Il Blocco di Mantova, l'anno scorso mi ha fatta esser modesta nella ricerca de' personaggi; ma per l'anno venturo, V. E. è in obbligo, e li corre per impegno di farmi una compagnia degna della sua elezione, con concedermi qualche personaggio che sin ora ho osato di chiedere, e che sin ora ho nutrito un genio particolare d'ottenere; e se taluno dei comici gode tal privilegio d'autorità assoluta, di levare e mettere qual personaggio gli piaccia, io non pretendo di conseguir nulla, se non mi viene dalle mani di V. E., ratificando di conoscerlo per protettore e per padre e niuno così certa delle grazie di V. E. che di più non posso esprimere. (ASMn, AG, E. XLV. 3, b. 1584) ) $^{13}$

Questa postulante non era che un'attrice, il signore era «protettore e padre». Da parte di lui non troviamo nessun rapporto affettivo o morale in questo affare, solo il pagamento di una prestazione d'opera che la decisione di un contabile o la preoccupazione del duca avrebbero realizzato. La Costantini lo sapeva bene, conosceva gli umori e le scelte aleatorie di Ferdinando Carlo: per questo motivo recitava il suo "personaggio" con forza. Pure, a fronte di un contratto già firmato, anche il duca doveva attendere. Nel 1702 Teresa comunicò che avrebbe potuto recarsi a Casale solo in primavera, soprattutto dopo che

li detti eccellentissimi Vendramin hanno fatto lo sborso di cinquecento e cinquanta ducati per ricuperare il nostro convoglio, $[\ldots]$ violenza cagionata dal poco utile ricavato nella città di Genova e di Lucca. [...] Per me non trovo forme con cui possa disimpegnarmi dagli Eccellentissimi Vendramin per due riflessi: il primo per aver essi sin da questa quadragesima passata, obbligata con scrittura la compagnia, e il secondo per aver fatto il sborso predetto di tanta conseguenza. (Ibidem) $)^{14}$

3. Nel 1702 Alvise Vendramin aveva scritturato un'altra indiscussa protagonista delle scene del tempo, Angela Paghetti, per recitare 'a vicenda' con Teresa Costantini, che così scriveva all'emissario gonzaghesco:

Il personaggio ricercato dall'Eccellentissimo Vendramin è la signora Paghetti, e perché finora stava dubbiosa la di lei venuta, per tale motivo io non l'ho

${ }_{13}$ Lettera di Teresa Corona Costantini a ignoto, Venezia, 7 ottobre 1702, cc. n.n. (largamente inedita, frammento in Selfridge-Field, 1998, p. 232).

${ }^{14}$ Lettera di Teresa Corona Costantini a ignoto, Venezia, 8 ottobre 1702, c. n.n. 
dichiarata nell'altra lettera; in questo ordine essa è comparsa e dovrà recitare nel di lui teatro a vicenda con la mia persona. Ed ora che tengo tale sicurezza, non tralascio di notificarli il mio contento nell'unione di tale meritevole comica, e per cui l'eccellentissimo Vendramin ha avuto tanto genio di conseguirla. Essa dunque è venuta a vicenda, ma perché la truppa è di già formata, ha convenuto alla detta signora portarsi a Venezia senza il di lei consorte, difficoltà che è stata l'unica da superarsi. (Ibidem) ${ }^{15}$

Esponente di una celebre famiglia d'Arte perché figlia della primadonna modenese Marzia Narici (Flaminia) e del napoletano Giuseppe Fiala (Capitano Sbranaleoni), la signora Angela nel 1686 figurava fra gli attori del duca di Mantova ospiti della compagnia ducale estense, beneficiaria del donativo di Francesco $\mathrm{II}^{16}$. Successivamente aveva ricoperto il ruolo di seconda donna nella troupe diretta da Agata Caterina Vitaliani Calderoni (Flaminia), capocomica di carriera internazionale ${ }^{17}$. Angela abitava a Bologna insieme a Giovanni Battista Paghetti (Dottore) e almeno otto figli ${ }^{18}$. Accettava il contratto veneziano da sola per assicurarsi uno stipendio e un protettore, dal momento che suo marito era stato recente oggetto di maldicenze che lo avevano screditato agli occhi del nobiluomo bolognese conte Isolani. Angela temeva una pena severa, come quando, trent'anni prima, fu segregata in un convento perché il consorte era stato sorpreso con lettere compromettenti (Cirani, 2004, p. 65):

15 Lettera di Teresa Corona Costantini a ignoto (Carlo Maria Vialardi?), Venezia, 7 ottobre 1702 , cc. n.n.

16 ASMo, ASE, Archivio per materie, Spettacoli pubblici, b. 8/A, ordine di pagamento di Francesco II d'Este, 16 marzo 1686, s.n.

17 Cfr. Ibidem, nota della compagnia, s.d. e s.n.

18 Giovanni Battista (Dottore), elogiato per non essere attore ignorante (Riccoboni, 1731, pp. 73-74 e Rasi, 1897-1905, II, pp. 203-204) ebbe Pietro (Dottore, poi Pantalone) scritturato nella troupe di Giuseppe Tortoriti (Pascarello), di cui sposò una figlia, alla foire di Parigi (1710-1714) e alla Comédie Italienne (1720-1732). Ferdinando Carlo lavorò con la madre nel ruolo di zanni. Giustina Francesca (Flaminia) fu dal 1713 nella compagnia «de los Trufaldines» col marito Domenico (Truffaldino) e il figlio Filippo Bononcini (Capitano Spagnolo), che divenne responsabile dei costumi delle opere italiane nella Real Tapiceria (Mémoire sur le Rétablissement, I, 1729, p. XX; Fuchs, 1976, pp. 19 e 162; Torrione, 2004, p. 788 e Morales, 2007, pp. 175 e 207). Una Margherita recitava probabilmente nel 1734 in Italia (Domenech Rico, 2007, p. 148). 
[...] la malignità di un suo avversario farlo decadere con indegni e falsi rapporti e con azzardo di farlo incorrere in qualche pericolo. Cotesto temerario maliziosamente ha fatto comparire il detto mio marito per un uomo non conoscitore della stima di un sì degno soggetto, e capace di quelle azioni di cui egli forse ha per proprio costume di maligno livore. In ciò s'estende la grazia, che in virtù della di lei autorevole protezione spero d'ottenere, e con tutta la dovuta rassegnazione de miei umilissimi rispetti, per sempre mi protesto, di Vostra Signoria Illustrissima, umilissima e devotissima serva, Angela Paghetti comica. (ASMn, AG, E. XLV. 3, b. 1584) $)^{19}$

Nel 1704 Angela dirigeva una compagnia accanto al Paghetti e al figlio Ferdinando Carlo nel ruolo di terzo amoroso. Pacata e più affidabile dell'imprevedibile Diana, la primadonna aveva stabilito con anticipo la programmazione in modo da assicurarsi gli attori migliori, aveva pianificato la tournée con calma, organizzando recite anche nelle tappe intermedie del viaggio ${ }^{20}$. Ma dopo lo smantellamento delle postazioni nel mantovano per spostare la guerra in Piemonte, di fatto i Francesi reggevano le sorti del ducato. Regnavano fame e disperazione e Angela, in buoni contatti col nord, firmò contratti per andare a recitare a Udine dopo Pasqua e a Brescia durante l'estate (Selfridge-Field, 1998, p. 239).

4. Nel febbraio 1704 l'impresario Vendramin aveva rinnovato il contratto a Colomba Coppa detta Aurelia, la cui troupe nel marzo venne richiesta al duca anche da Giovan Carlo Grimani per il San Samuele ${ }^{21}$. Aurelia risiedeva da molti anni a Ferrara, dove aveva preso in affitto

19 Lettera di Angela Paghetti a ignoto, Venezia, 9 dicembre 1702, c.n.n.

20 Cfr. «Angela Paghetti, prima donna, Camilla Bissoni, seconda donna, Rosa Nobili, serva, Leandro Possi, primo moroso, Santo Nobili, secondo moroso, Carlo Paghetti, terzo moroso, Gio[vanni] Gagi Pantalone, Dottor Paghetti, Stiuono primo zanni, Gradelino o sia Carlo Rusca secondo zanni» (ASMn, AG, E. XXXI. 3, b. 1277, compagnia di Angela Paghetti, <1704?>, c. n.n.).

21 «Aggiongo inoltre le mie rispettosissime istanze che volendosi V. A. S. servire d'essa compagnia in Carnevale si compiaccia in tal caso concedermi la compagnia di Diana [...]» ASMn, AG, E. XLV. 3, b. 1585, lettera di Giovan Carlo Grimani a Ferdinando Carlo Gonzaga-Nevers, Venezia, 15 marzo 1704, cc. n.n. 
quattro camerette di proprietà del duca di Modena ${ }^{22}$. Scritturata nel ruolo di seconda donna nella compagnia delle sorelle Antonia e Angiola Isola (Lavinia e Leonora) sotto il patrocinio di Francesco II d'Este ${ }^{23}$, nel 1702 era passata sotto l'egida di Ferdinando Carlo col marito Giuseppe detto Virginio (poi Trinella) e il figlio Antonio. Recitava in una prima parte probabilmente "a vicenda" con Isabella Costantini Servilli detta Eularia $^{24}$, "comica virtuosissima del Serenissimo di Mantova, in rappresentare in Bologna le metamorfosi di Eularia, finta lacchè francese, dama spagnola, soldato tedesco, e cingara egittiaca, con spacchi di spadone, alabardino, due spade, scherma nell'Academia degli esercizi militari, e con quattro suoi balletti diversi ${ }^{25}$. La fama di Eularia si evince dal suo contratto veneziano di settecento ducati anziché gli abituali cinquecento $^{26}$. Potrebbe essere lei, sorella di Giovanni Battista Costantini, che recitò "a vicenda" anche con la cognata Teresa detta Diana e che, insieme al marito Francesco Servilli detto Odoardo, fu protagonista degli spettacoli mantovani nell'autunno 1701 e nell'estate 1702 (Cirani, 2004, pp. 209-210).

Nell'aprile 1704, quando Ferdinando Carlo era a Parigi per concordare nuove nozze con Luigi XIV dopo la morte della moglie Anna Isabella, la compagnia di Colomba Coppa detta Aurelia si trovava a Milano. Dopo un viaggio accidentato e la fredda accoglienza dell'impresario Antonio Piantanida, la capocomica si era ripromessa di non mettere più piede in quel teatro e ne scriveva al duca. L'offerta del Grimani a Venezia arrivava a proposito. Tuttavia l'amministratore del San Samuele, Nane Piccolo, aveva proposto un contratto con paga ridotta di un quarto e metà della cifra in regalo. In più non era previsto alcun anticipo. Ricor-

22 Cfr. ASMo, ASE, b. unica Comici, fasc. Coppa Giuseppe detto Virginio artista comico 1689-1692, supplica di Giuseppe e Colomba Coppa a Francesco II d'Este, Ferrara, 27 aprile 1691, c. n.n.

23 Ibidem, nota della prima compagnia de' comici, s.l. e s.d., c.n.n.

24 Cfr. ASMn, AG, E. XLV. 3, b. 1584, lettera di Teresa Corona Costantini a ignoto, Venezia, 7 ottobre 1702 , cc. n.n.

25 Dedica al sonetto anonimo di lode Questa è la saggia Eularia e questa è quella, trascritti in Rasi, 1897-1905, III, p. 533.

26 Accordi del 1 marzo 1705 riprodotti in Alberti, 1987, p. 147. 
dando che due anni prima Diana aveva ricevuto i suoi cinquanta ungari pur avendo recitato solo mezza stagione, Aurelia paventava la chiusura della compagnia e così spiegava al duca:

Il punto è questo, Serenissimo Padrone, e V. A. S. comandi o servire all'Eccellentissimo Vendramin sì benemerito verso i servitori di V. A. S. con esborsi grandi, anticipati, interi, con mantenimento di cassetta, con alloggi nobilissimi, con provvigioni di piazze, avendo già procurato l'assenso dagli eccellentissimi rettori di Brescia e Bergamo per una compagnia che resterà da V. A. S. destinata in San Luca; ovvero a Nane Piccolo, che ci ha riconosciuti con le sopradette maniere, che vuol pareggiar le condizioni proposte da V. A. S., che non vuol soccorrerci di presente, che non vuol mantenerci l'utile della cassetta. V. A. S. vede gl'utili d'un partito, i pregiudizi dell'altro, noi siamo suoi servitori che sempre abbiamo avuto ogni nostro essere dalla protezione di V. A. S. quale in questa congiuntura è pregata di carità e alla quale baciamo la veste [...]. (ASMn, AG, E. XLV. 3, b. 1585) ${ }^{27}$

La compagnia venne messa sotto contratto per il San Luca con anticipo pagato e il solito compenso ${ }^{28}$, mentre Aurelia avrebbe avuto un aumento di paga da seicento a novecento quattro ducati ${ }^{29}$. La capocomica accettò di buon grado i numerosi regali di Ferdinando Carlo - bicchieri, cinque pettini e gioielli ${ }^{30}$ - ma a metà marzo fece sapere che non avrebbe più recitato perché le spettavano altri duecento ducati ${ }^{31}$, e che per l'anno successivo si sarebbe spostata a Padova oltre che in Laguna ${ }^{32}$.

27 Lettera di Colomba Coppa, Giovanna Benozzi Sassi e Giovan Battista Sassi a Ferdinando Carlo Gonzaga-Nevers, Venezia, 22 marzo 1704, c. n.n.

28 Ivi, copialettera dell'impegnativa di scrittura teatrale di Alvise Vendramin per la compagnia di Colomba Coppa, Venezia, 20 febbraio 1704, c. n.n.

${ }^{29}$ Cfr. ivi, lettera di Francesco e Alvise Vendramin a ignoto, Venezia, s.d. (marzo 1704?), cc. n.n.

30 ASMn, AG, E. XLV. 3, b. 1587, lettera di Colomba Coppa a ignoto, Venezia, 7 febbraio 1705 , c. n.n.

31 Cfr. ASMn, AG, E. XLV. 3, b. 1586, lettera di Colomba Coppa a ignoto, Venezia, 14 marzo 1705, c. n.n.; ivi, lettera di Giovan Carlo Grimani a ignoto, Venezia, 24 marzo 1705 , c. n.n.

32 Cfr. ivi, lettera di Pietro Rubinati a ignoto, Venezia, 28 marzo 1705, c. n.n. e lettera di Colomba Coppa a ignoto, Venezia 7 aprile 1705, c. n.n. 
Nel dicembre 1705 Ferdinando Carlo promise all'impresario Vendramin che «per l'autunno e Carnevale dell'anno prossimo venturo procurerò di darle una delle migliori mie compagnie dei comici sapendo quanto sia loro di profitto cotesto suo teatro» ${ }^{33}$. Nonostante la situazione politica irreparabilmente compromessa, nel 1706 il Gonzaga-Nevers pieno di debiti, ma per nulla rovinato ${ }^{34}$ - dava incarico all'architetto Ferdinando Galli Bibbiena di costruire un nuovo teatro ducale a Mantova (Amadei, 1973, p. 76).

5. Gli ultimi anni di Ferdinando Carlo, condannato per fellonia, segnano anche la fine delle sue splendide compagnie. Un mese dopo la battaglia di Torino, a Teresa Costantini non era rimasta che «la truppa più disgraziata del mondo» e il conte Muzio Francesco Cremona, residente mantovano, minacciava seri provvedimenti. Era anche in conseguenza delle sue bizze che la capocomica aveva perso cinque «personaggi», passati alla troupe concorrente. A eccezione del primo e del secondo amoroso (Girolamo Ferrari detto Silvio e Giuseppe Monti detto Odoardo), Diana aveva dovuto ripiegare su attori improvvisati: il suggeritore nel ruolo del Dottore, due fratelli musicisti come primo zanni e terzo amoroso, la moglie di quest'ultimo a fare la servetta; secondo zanni Giuseppe Roffi, cantimbanco sul mercato di Reggio.

Questi sono cinque personaggi acquistati da me nelle mie infelicità a costo della mia miserabile borsa vestiti, et alimentati per procacciarle la fortuna, e che attualmente mi vanno debitori di bona somma di denaro, sì anche di due sopradetti che se ne andarono in Bologna. [...] stante dunque li sopradetti sincerissimi punti e significati all'A.V.S., mi permetta la di lei clemenza che possa supplicarla a riflettere esser verso di me troppo rigoroso il predetto signor Conte Cremona nel minacciarmi la disgrazia dell'A.V.S. quando io in conto alcuno non ho mancato e sono pronta a obbedire interamente a quanto l'A.V.S. vorrà obbligarmi, mentre io ho adempito a quello che l'Ec-

33 ASVe, Inquisitori di Stato. Case da Gioco Teatri, b. 914, fasc. Teatro S. Lucca, copia coeva del biglietto di Ferdinando Carlo Gonzaga-Nevers a Alvise Vendramin, Casale, 23 dicembre 1704 (more veneto), c. n.n.

34 Cfr. i beni inventariati alla morte del duca (contanti, gioielli e pietre preziose, 299 cavalli, quadreria di circa novecento pezzi) in Malacarne, 2009, pp. 301-303. 
cellentissimo Grimani mi scrisse, tanto più che realmente li predetti cinque personaggi spontaneamente andiedero ad unirsi otto giorni prima della mia partenza da Bergamo, con la detta Paghetti, e porsi (?) per recitare in Crema nonostante che mi dichiarassi che bisogna attendere le dichiarazioni della volontà di V. A. S. [...] et se non credessi che riuscisse troppo temerario la mia esposizione direi a V. A. S. che in oggi sono con un corpo di compagni che esigono dalla città di Venezia tanto compatimento che in quattro recite che abbiamo fatto abbiamo avuto (non per le qualità mie) ma per ciaschedun altro di compagni tanta fortuna che il teatro di S. Luca ha invidiato interamente il concorso che abbiamo avuto. (ASMn, AG, E. XLV. 3, b. 1586)

Nell'inverno 1707 Ferdinando Carlo si ritirò a Venezia, mentre la moglie Susanna Enrichetta di Lorena partì definitivamente per la Francia. Nel marzo, dopo l'occupazione austriaca di Mantova, i musicisti del duca furono esortati dall'imperatore a dare le dimissioni (SelfridgeField, 1998, pp. 242-243). Anche Carlo Maria Vialardi, fedelissimo del Gonzaga, si mise a disposizione dell'amministrazione asburgica. La scomparsa di Ferdinando Carlo come mecenate di cantanti e di attori rese necessaria la riorganizzazione della vita teatrale. Angela Paghetti, ormai avanti con gli anni, probabilmente pensò al ritiro. Colomba Coppa non si sganciò dal patrocinio mantovano: nel marzo 1707 le fu rinnovato il contratto ${ }^{36}$, ma già in aprile scrisse che non era stata pagata e aveva fame ${ }^{37}$. Teresa Costantini tornò a firmarsi «comica del duca di Parma» e a Milano, «per guadagnarmi una gran protezione» ${ }^{38}$, dedicò al comandante dell'armata imperiale e cavaliere del Toson d'Oro, conte Wirrico di Daun, l'edizione a stampa de La casta Penelope di cui era applaudita protagonista. L'autore Pietro Pariati le aveva cucito addosso un ruolo assai patetico, il simbolo per antonomasia della costanza e della fedeltà.

${ }^{35}$ Lettera di Teresa Corona Costantini a Ferdinando Carlo Gonzaga Nevers, Venezia, 16 ottobre 1706 , cc. n.n.

${ }^{36}$ Andrea, Francesco e Alvise Vendramin, contratto di scrittura teatrale, Venezia, 24 marzo 1707 in Alberti, 1987, p. 145.

${ }^{37}$ Lettera di Colomba Coppa a ignoto, Venezia, 2 aprile 1707, citata in SelfridgeField, 1998, p. 243.

${ }^{38}$ Dedica di Teresa Costantini in Pariati, 1707, s.n. 
Lista delle abbreviazioni bibliografiche:

AG - Archivio Gonzaga

ASE - Archivio Segreto Estense

ASMn - Archivio di Stato di Mantova

ASMo - Archivio di Stato di Modena

ASVe - Archivio di Stato di Venezia

\section{BIBLIOGRAFIA}

Alberti, C. (1987). Il ritorno dei Comici. Vicende del Teatro Vendramin di San Luca (1700-1733). Biblioteca Teatrale, 5-6, 13-187.

Alberti, C. (1990). La scena veneziana nell'età di Goldoni. Roma: Bulzoni.

Amadei, G. (1973). I centocinquant'anni del Sociale nella storia dei teatri di Mantova. Mantova: Citem.

Bartoli, F. (1781-1782). Notizie istoriche de' comici italiani che fiorirono intorno all'anno MDL. Fino a' giorni presenti, 2 voll. Padova: Conzatti (online a cura di G. Sparacello, «Les savoirs des acteurs italiens», 2010. Retrieved from www.iremus.cnrs.fr.

Benzoni, G. (1996). Ferdinando Carlo Gonzaga-Nevers. In Dizionario Biografico degli Italiani (vol. 46, pp. 283-284). Roma: Istituto della Enciclopedia Italiana.

Besutti, P. (1989). La corte musicale di Ferdinando Carlo Gonzaga ultimo duca di Mantova. Musici, cantanti e teatro d'opera tra il 1665 e il 1707. Mantova: Arcari.

Bianchi, A. (2012). Al servizio del principe. Diplomazia e corte nel ducato di Mantova 1665-1708. Milano: Unicopli.

Borrelli, A. (2002). Vincenzo Grimani. In Dizionario Biografico degli Italiani (vol. 59, pp. 668-562). Roma: Istituto della Enciclopedia Italiana.

Carpeggiani, P. (2005). Spazi teatrali nel Palazzo Ducale di Mantova. In N. Zuccoli (Ed.), Teatri storici nel territorio mantovano. Forme, significato, funzioni (pp. 37-60). Mantova: Arcari.

Cirani, P. (2004). Comici, musicisti di teatro alla corte di Ferdinando Carlo Gonzaga Nevers. Mantova: Casa del Mantegna. 
Domenech Rico, F. (2007). Los Trufaldines y el teatro de Los Caños de Peral. La Commedia dell'Arte en la España de Felipe V. Madrid: Fundamentos.

Fantini D’Onofrio, F. (1988). Le fonti e la storia. La guerra di successione spagnola a Mantova attraverso la corrispondenza ai Gonzaga da Mantova e Paesi. In C. Belfanti, F. Fantini D’Onofrio \& D. Ferrari (Eds.), Guerre Stati e Città. Mantova e l'Italia padana dal sec. XIII al XIX (pp. 427-466). Mantova: Arcari.

Fochessati, G. (1930). I Gonzaga di Mantova e l'ultimo duca. Milano: Ceschina.

Fuchs, M. (1976). La vie théâtrale en province au XVIIIe siècle. Lexique des troupes de comédiens au XVIIIe siècle. Genève: Slatkine Reprints.

Maffi, D. (2010). La cittadella in armi. Esercito, società e finanza nella Lombardia di Carlo II 1660-1700. Milano: Angeli.

Malacarne, G. (2008). I Gonzaga di Mantova: una stirpe per una capitale europea, vol. 5. Modena: Il bulino.

Mancini, F., Muraro, M.T., \& Povoledo, E. (Eds.). (1995). I teatri del Veneto, t. 1: I teatri di Venezia. Teatri effimeri e nobili imprenditori. Venezia: Regione del Veneto-Giunta Regionale-Corbo e Fiore.

Mémoire sur le Rétablissement des Comédiens Italiens à Paris (1729). In Nouveau Théâtre Italien, ou recueil général des Comédies représentées par les Comédiens Italiens Ordinaires du Roi. Augmentée des Pièces nouvelles, des Arguments de plusieurs autres qui n'ont point été imprimées, et d'un Catalogue de toutes les Comédies représentées depuis le rétablissement des Comédiens Italiens, vol. 1. Paris: Briasson.

Morales, N. (2007). L'artiste de cour dans l'Espagne du XVIII siècle: Étude de la communauté des musiciens au service de Philippe V (1700-1746). Madrid: Casa de Velázques.

Morinello, C. (1956). Costantini. In Enciclopedia dello Spettacolo, vol. 3 (coll. 1568-1569). Roma: Le Maschere.

Muratori, L.A. (1827). Annali d'Italia dal principio dell'età volgare sino all'anno MDCCXLIX, compilati da L. A. Muratori e continuati sino all'anno 1827, vol. 27. Firenze: Marchini.

Pariati, P. (1707). La casta Penelope. Milano: Ghisolfi.

Rasi, L. (1897-1905). I Comici italiani. Biografia, bibliografia, iconografia, 3 voll. Firenze: Lumachi.

Ricci, C. (1888). I teatri di Bologna nei secoli XVII e XVIII. Storia aneddotica. Bologna: Monti (rist. anast. 1965. Bologna: Forni). 
Riccoboni, L. (1731). Histoire du Théâtre Italien depuis la décadence de la comédie latine; avec des Extraits, et Examens critiques de plusieurs tragédies, et comédies italiennes, auxquels on a joint une explication des figures, avec une Lettre de M. Rousseau, et la Réponse de l'Auteur, 2 voll. Paris: Cailleau.

Selfridge-Field, E. (1985). Pallade Veneta. Writings on Music in Venetian Society 1650-1750. Venezia: Fondazione Levi.

Selfridge-Field, E. (1998). «La Guerra de' comici»: mantuan comedy and venitian opera in ca. 1700. Recercare, X, 208-248.

Torrione, M. (2004). «Como a vuestra majestad le gustan las comedias». Felipe V y la compañia de los Trufaldines: 1703-1725. In E. Serrano (Ed.), Felipe Vy su tiempo, Congreso Internacional (t. II, pp. 753-789). Zaragoza: Instituto Fernando El Católico.

Zaccaria, M. (2018). Una famiglia d'Arte fra Sei e Settecento: i Costantini. Biblioteca Teatrale, 127-128 (parte prima), 65-80.

Riassunto: Il contributo presenta l'attività delle capocomiche Teresa Costantini, Angela Paghetti, Colomba Coppa al servizio di Ferdinando Carlo Gonzaga-Nevers, ultimo duca di Mantova e generoso mecenate. Sulla linea di studi iniziati trenta anni fa sul versante musicologico e delle recenti ricerche storiche, il saggio, condotto su fonti d'archivio, mette in evidenza le responsabilità di ordine artistico, organizzativo e gestionale delle capocomiche della Commedia dell'Arte e ricostruisce da un lato i loro rapporti con Ferdinando Carlo, gli agenti ducali e la corte gonzaghesca; dall'altro con gli impresari veneziani Grimani, con i quali il duca di Mantova aveva stabilito una duratura e generosa collaborazione.

Le donne di spettacolo, pregiudizialmente equiparate alle prostitute e beneficiarie di regali e di privilegi, erano raffigurazioni d'amore e di gloria per un principe screditato, la cui schiera di artisti fu però fra le più folte e dinamiche d'Italia. Le attrici si allontanarono progressivamente dai codici della cortigianeria e in qualità di professioniste scritturate e retribuite in denaro vantarono forte potere contrattuale e margini di autonomia; ma furono sottoposte a regole e obblighi soprattutto a Venezia, dove i proprietari dei teatri rendevano disponibili gli spazi solo alle compagnie sotto la protezione dei prìncipi politicamente allineati con la Serenissima.

Durante gli anni della cosiddetta guerra di successione spagnola, Diana, Aurelia e le altre si mossero fra Venezia, Mantova e Casale, dove Ferdinando Carlo si era trasferito dopo il blocco di Mantova accompagnato da un variopinto corteggio di donne, musicisti e commedianti. Le disavventure teatrali si incrociarono con le manovre militari nel territorio mantovano e dietro i cambi di destinazione, di programma o di repertorio leggiamo in filigrana la storia di un ducato che aveva smarrito la propria identità e di un mercato teatrale in rapida trasformazione.

Parole chiave: attrice, Commedia dell'Arte, Ferdinando Carlo Gonzaga-Nevers, capocomica, mercato teatrale 